\title{
Ceftazidime-Avibactam for Hospital Acquired Pneumonia Due to Extended Drug-Resistant Klebsiella pneumoniae
}

\author{
Amit Ranjan Rup ${ }^{1}$ (D) - Arun Kumar Dash ${ }^{1}$ (D) Sibabratta Patnaik ${ }^{1}$ (D) \\ Received: 14 August 2020 / Accepted: 14 October 2020 / Published online: 21 October 2020 \\ (C) Dr. K C Chaudhuri Foundation 2020
}

To the Editor: Emergence of extensive drug resistant and pan drug resistant Klebsiella pneumoniae (XDR-KP and PDR$\mathrm{KP}$ ) as nosocomial infection in Pediatric Intensive Care Unit (PICU) and treating these cases, is challenging for pediatricians as fewer data are available [1].

A 3-y-old boy was admitted to PICU in status epilepticus with history of sudden onset headache followed by generalized seizures without any fever. In view of low Glasgow coma scale (GCS), he was intubated and ventilated. MRI angiogram showed ruptured arterio-venous malformation. Initial laboratory parameters were normal. On third day, he developed high grade fever with increased ventilator requirement; tests were suggestive of sepsis, pneumonia, leucopenia and severe thrombocytopenia. Antibiotics were upgraded from ceftriaxone to meropenem and colistin was added after $2 \mathrm{~d}$. Both blood and endotracheal tube culture showed KP resistant to all antibiotics, except fosfomycin. Child continued to be critical with progressive leucopenia and thrombocytopenia; film array (multiplex-PCR) of endotracheal secretion showed KP with presence of CTX-M (Cefotaxime), NDM (New Delhi Metallo- $\beta$-lactamase) and OXA-48 like (Oxacillinase). Child was treated with inj. ceftazidime-avibactam $(62.5 \mathrm{mg} / \mathrm{kg} / \mathrm{dose}$ 8 hourly), inj. aztreonam and inj. fosfomycin. Child was isolated; extubated and invasive lines were removed. After $14 \mathrm{~d}$ of antibiotics, he was discharged with normal clinical and laboratory parameters. On active surveillance, same organism was cultured from emergency department suction bottle and MRI bed. Suction bottle disinfection was done with $1 \%$ hypochlorite solution and staff were trained for reprocessing of suction bottle. As a policy, the MRI bed is cleaned with alcohol and glutaral based disinfectant after each patient.

Sibabratta Patnaik

drsbpatnaik45@gmail.com

1 Department of Pediatrics, Kalinga Institute of Medical Sciences, Bhubaneswar, Odisha 751024, India
Ceftazidime-avibactam has excellent activity against many extended spectrum $\beta$-lactamase, Amp-C, KP-carbapenemase and OXA-48 producing enterobacteriaceae; however it is not active against NDM strains [2]. Aztreonam is effective against NDM strains as NDM cannot hydrolyse aztreonam. Literature suggests combination of ceftazidime-avibactam, fosfomycin and aztreonam; as survival in combination therapy is better [3]. Avibactam inhibits the KP carbapenemase (KPC) enzyme, ensuring not only the activity of ceftazidime but also that of aztreonam. A potential synergistic effect in this $\beta$ lactam combination is also expected, as both ceftazidime and aztreonam binds to PBP3 [4]. Ceftazidime-avibactam is recently approved by USFDA for treating children with complicated urinary tract infection and intra abdominal infection. Literature is scarce on use of ceftazidime-avibactam in pediatric hospital acquired pneumonia as safety is not established. However, due to rapid deterioration of our patient, it was used as a salvage therapy. Presently, multicentric clinical trial is going on to assess the safety and tolerability of ceftazidimeavibactam in nosocomial pneumonia [5].

Ceftazidime-avibactam stands out as one of the most important additions in our armamentarium for management of XDR and PDR-KP infection.

\section{Compliance with Ethical Standards}

Conflict of Interest None.

\section{References}

1. Krapp F, Ozer EA, Qi C, Hauser AR. Case report of an extensively drug-resistant Klebsiella pneumoniae infection with genomic characterization of the strain and review of similar cases in the United States. Open Forum Infect Dis. 2018;5:ofy074.

2. Iosifidis E, Chorafa E, Agakidou E, et al. Use of ceftazidimeavibactam for the treatment of extensively drug-resistant or pan drug-resistant Klebsiella pneumoniae in neonates and children $<5$ years of age. Pediatr Infect Dis J. 2019;38:812-5. 
3. European Centre for Disease Prevention and Control. Outbreak of carbapenemase-producing (NDM-1 and OXA-48) and colistinresistant Klebsiella pneumonia ST307. North-East Germany, 2019. 28 October 2019. ECDC: Stockholm. 2019.

4. Camargo JF, Simkins J, Beduschi T, et al. Successful treatment of carbapenemase-producing pandrug-resistant Klebsiella pneumoniaen bacteremia. Antimicrobial Agents Chemother. 2015;59:5903-8.

5. Pfizer. A Phase I, Open-Label, Single-dose Study to Assess the Pharmacokinetics, Safety and Tolerability of Ceftazidime-
Avibactam (CAZ-AVI) in Children from 3 months to less than 18 years of age who are Hospitalized and Receiving Systemic Antibiotic Therapy for Suspected or Confirmed Nosocomial Pneumonia including Ventilator Associated Pneumonia. Available at: https:/clinicaltrials.gov/ct2/show/NCT04040621. NLM identifier: NCT04040621. Accessed August 13, 2020.

Publisher's Note Springer Nature remains neutral with regard to jurisdictional claims in published maps and institutional affiliations. 